\title{
Castiglione à la cour de Charles Quint. La langue et les pratiques de la diplomatie italienne à la Renaissance
}

Castiglione alla corte di Carlo V. Lingua e procedure della diplomazia italiana nel Rinascimento

Castiglione at the Court of Charles V. Language and Practice of Renaissance Italian Diplomacy

\section{Raffaele Ruggiero}

\section{OpenEdition} Journals

Édition électronique

URL : http://journals.openedition.org/cei/5239

DOI : $10.4000 /$ cei.5239

ISSN : 2260-779X

\section{Éditeur}

UGA Éditions/Université Grenoble Alpes

\section{Édition imprimée}

ISBN : 978-2-37747-063-1

ISSN : 1770-9571

\section{Référence électronique}

Raffaele Ruggiero, «Castiglione à la cour de Charles Quint. La langue et les pratiques de la diplomatie italienne à la Renaissance », Cahiers d'études italiennes [En ligne], 27 | 2018, mis en ligne le 30 septembre 2018, consulté le 26 mars 2021. URL : http://journals.openedition.org/cei/5239 ; DOI https://doi.org/10.4000/cei.5239

Ce document a été généré automatiquement le 26 mars 2021. 


\title{
Castiglione à la cour de Charles Quint. La langue et les pratiques de la diplomatie italienne à la Renaissance
}

\author{
Castiglione alla corte di Carlo V. Lingua e procedure della diplomazia italiana nel \\ Rinascimento \\ Castiglione at the Court of Charles V. Language and Practice of Renaissance \\ Italian Diplomacy
}

Raffaele Ruggiero

1 Castiglione est bien connu dans la culture européenne en tant qu'auteur du Courtisan, le dialogue en quatre livres paru en 1528, juste avant sa mort à Tolède, lorsqu'il était «nonce », c'est-à-dire ambassadeur permanent du pape en Espagne, auprès de la cour de l'empereur Charles Quint. Ce livre, auquel l'auteur avait consacré presque toute sa vie littéraire, se propose d'offrir un portrait du courtisan parfait à travers un dialogue qui se déroule à la cour de Guidubaldo da Montefeltro, duc d'Urbino, où Castiglione, pendant sa jeunesse et au début de sa carrière diplomatique, avait été parmi les fonctionnaires les plus actifs ${ }^{1}$. Malgré les doutes et les incertitudes de Castiglione, le livre du Courtisan connut un très vif succès : rééditée et réimprimée plusieurs fois en italien, l'œuvre fut très rapidement traduite en espagnol (1534), en français (1537), en anglais (1561), en allemand et en latin. Le livre devint le manuel de formation de l'élite dirigeante européenne pendant l'Ancien Régime².

2 En effet, la réception du Courtisan est marquée par la même ambiguïté qui distingue la carrière diplomatique de Castiglione : l'ambassadeur du pape (auprès de la cour d'un empereur comme Charles Quint, lorsqu'il décide de proposer un portrait du courtisan parfait et obligatoirement aussi d'une cour parfaite), nous amène dans une toute petite cour de l'Italie centrale, qui est dépourvue de pouvoir effectif, la cour d'un duc malade et à la recherche d'appui. Il faut souligner l'idée que le Courtisan est un portrait, une 
image de la cour et de ses fonctionnaires, parce que c'est exactement comme cela que Castiglione présente son livre dans la dédicace à l'évêque portugais Miguel de Sylva :

[...] un ritratto di pittura della corte d'Urbino, non di mano di Raphaello o Michel'Angelo, ma di pittor ignobile e che solamente sappia tirar le linee principali, senza adornar la verità de' vaghi colori o far parer per arte di prospettiva quello che non $\grave{\mathrm{e}}^{3}$.

C'est justement dans la "perspective » que se révèlera véritablement le projet culturel de Castiglione.

3 Le livre est marqué par le concept de distance: la distance temporelle, entre l'expérience de jeunesse de Castiglione à Urbino et sa tâche diplomatique à la cour impériale ; la distance spatiale, entre la petite ville d'Urbino et la scène de la politique internationale dans l'Espagne de Charles Quint. À cela s'ajoute enfin la distance entre les niveaux d'action possible dans la dialectique des petits États régionaux italiens entre la fin $d u x^{e}$ et le début $d u x v I^{e}$ siècle et les relations diplomatiques continentales qui se déroulent entre l'Empire, la France de François I $^{\text {er }}$ et l'Angleterre d'Henri VIII, avec les tensions religieuses et politiques causées par la Réforme en Allemagne et par l'avancée des Turcs en Europe orientale. De plus, la stratégie du livre est caractérisée par l'idée d'absence : le dialogue se déroule à la cour d'Urbino, lorsque Castiglione n'y est pas. En fait, en 1506, il fut envoyé en Angleterre en tant que procureur du duc d'Urbino, afin de recevoir à sa place les enseignes de chevalier de la Jarretière (il s'agit $\mathrm{du}$ tout début de la carrière diplomatique internationale de Castiglione $)^{4}$. D'ailleurs, le livre est publié en Italie, par la maison d'édition européenne la plus éminente, celle des héritiers d'Aldo Manuzio, le père des imprimeurs humanistes. Par ailleurs, le livre est imprimé lorsque l'auteur est en Espagne, absent et lointain, et il est confié aux soins des hommes de lettres les plus illustres de son temps ${ }^{5}$.

4 Ces idées de distance et d'absence sont en effet la clé d'interprétation de la diplomatie italienne au début du XVI ${ }^{e}$ siècle, lorsque la politique italienne se fonde entièrement sur la pratique de la parole, et donc sur la rhétorique, à cause de l'impuissance des petits États régionaux italiens par rapport aux grandes monarchies européennes. Le livre du Courtisan ne doit alors plus être considéré en tant que modèle, comme un manuel de formation et de comportement pour les hommes d'État, mais plutôt comme la tentative de donner une réponse à la crise de la diplomatie italienne, une stratégie pour abandonner des pratiques politiques devenues irréalisables: le Courtisan est le diagnostic d'une maladie expérimentée par l'auteur pendant sa propre activité diplomatique, en même temps qu'il est la proposition d'un remède. Ce remède pourra éventuellement se révéler aussi efficace après le sac de Rome, lorsque l'espace politique italien n'est plus praticable en tant que tel, et quand l'Italie n'est plus l'espace d'une politique nationale ${ }^{6}$.

5 La carrière diplomatique de Castiglione débuta - on l'a dit - en 1506, avec la mission en Angleterre en tant que procureur du duc d'Urbino, ce dernier ayant été nommé par Henri VII chevalier de la Jarretière. Dans cette phase de sa vie et de sa carrière, Castiglione est un fonctionnaire de la cour d'Urbino et de la cour de Mantoue, notamment en relation de collaboration avec les duchesses Isabella d'Este Gonzague et Elisabeth Gonzague. Son activité diplomatique est également partagée entre les cérémonies d'apparat et des missions délicates et de plus en plus importantes dans le cadre des équilibres politiques européens. Juste avant la bataille de Ravenne, en mars 1512, il fut chargé d'une mission secrète à Blois, auprès de Louis XII, une mission conduite grâce à l'appui du trésorier Florimond de Robertet, dans laquelle le duc 
d'Urbino promettait d'abandonner le pape (son oncle) et de s'allier avec la France. Juste après la bataille, Castiglione rédigea la lettre dans laquelle le duc d'Urbino annonçait à son beau-père, le marquis de Mantoue, qu'il était en train d'abandonner l'alliance avec la France afin de retourner dans l'armée pontificale. Par la suite, pendant le pontificat de Jean de Médicis (Léon X), Castiglione fut l'ambassadeur permanent du marquis de Mantoue à la cour de Rome ${ }^{7}$. Pendant cette dernière mission, Castiglione mûrit les deux principes porteurs de sa ligne politique : une alliance entre le pape et les petits États régionaux de l'Italie centrale et septentrionale, en considérant l'État de l'Église comme le seul capable de mener une politique presque " nationale » au même niveau que celle des autres monarchies européennes; et une alliance entre le pape et l'empereur, à même d'assurer l'indépendance des territoires italiens septentrionaux, qui étaient, jusqu'à ce moment-là, des otages des guerres franco-espagnoles ou franco-impériales ${ }^{8}$. Un choix politique précis avait orienté l'action de Castiglione pendant les premières années $\mathrm{du} \mathrm{xvI}{ }^{\mathrm{e}}$ siècle, lorsque les petits États régionaux italiens furent écrasés par les guerres d'équilibre entre les grandes monarchies continentales: il avait très tôt reconnu que seul l'État de l'Église pouvait jouer un rôle presque égal avec les autres États nationaux, en raison de son ascendant spirituel et de son organisation bureaucratique. Seuls les princes ecclésiastiques

[...] hanno stati e non gli difendono; hanno sudditi e non li governano. E gli stati, per essere indifesi, non sono loro tolti; ed e' sudditi, per non essere governati, non se ne curano, né pensano né possono alienarsi da loro. Solo adunque questi principati sono sicuri e felici'.

Et dans l'orbite de la politique pontificale devait rentrer l'action de ses satellites les plus proches, en marquant une fois de plus le déplacement de l'effectif pouvoir décisionnel à Rome.

Grâce à sa position politique, bien connue quoique minoritaire auprès de la cour pontificale (où le parti le plus puissant, auquel appartenaient Guichardin, le "datario » Giberti, ainsi que l'habile Alberto Pio de Carpi, favorisa l'alliance avec la France de François $\mathrm{I}^{\mathrm{er}}$ ), à la fin de 1524, Castiglione fut nommé nonce pontifical auprès de la cour impériale de Charles Quint en Espagne.

Pendant le mois de mars 1526, la négociation pour la paix entre l'Empire et la France, et la libération de François $\mathrm{I}^{\mathrm{er}}$ (qui avait été fait prisonnier après la bataille de Pavie), arrive à ses dernières phases: la cour impériale est divisée, mais la plupart des diplomates semblent convaincus que la France ne respectera pas ses promesses. Dans ce cadre, le chancelier Mercurino Arborio de Gattinara va jusqu'à refuser de signer le traité avec le sceau de la chancellerie. Le chancelier manifeste sa propre autonomie par rapport à l'orientation politique de l'empereur, en soulignant qu'il est obligé de ne rien faire qui puisse nuire à l'empereur :

E a questi dì passati volendo l'Imperatore che 'l Cancelliero sottoscrivesse l'accordo di Francia con S. M., il Cancelliero rispose che non volea farlo, perché secondo l'officio suo era obbligato a non far cosa ch'ei conoscesse o giudicasse in sua coscienzia che venisse a danno di S. M., ancora che quella glielo comandasse. L'Imperatore soggiunse che se fosse cosa dove andasse interesse del Papa, che non se ne farebbe pregare; e 'l Cancelliero gli rispose che S. M. s'ingannava, perché egli non avea l'occhio ad altro che al servizio di quella: ben le protestava che per modo alcuno non volea più accettare il Cardinalato, e che manderebbe i sigilli a $\mathrm{S}$. M. acciò ch'ella ne facesse il suo parere. Replicò l'Imperatore che se glieli mandava non glieli restituirebbe più. Il Cancelliero gli disse che in questo riceveria grazia da S. M., ma che egli non volea per modo alcuno far quello, che non si conveniva all'officio suo. In questo parve l'Imperatore si mitigasse [...]. La cosa restò così; e come ho detto 
l'Imperatore s'acquetò, dicendo che ben sapea di non gli aver fatto quelle mercedi che meritava, ma che le farebbe. [...] Il gran Cancelliero fa l'officio suo con la medesima autorità, e dice pubblicamente che crede che il re di Francia non manterrà le promesse. [...]

El ragionamento che Mons. Rev.mo Legato et io havessimo con l'Imperatore [...] fu alli 18 del presente [...], e mostra $\mathrm{S}$. Maestà credere che 'l Cristianissimo mantenerà quel che ha promesso. Dice haver ditto a lui proprio che quel de loro che saltarà a l'altro, havrà da esser ragionevolmente tenuto mechant uomo e velliacho. Dissemi el Confessor che S. Maestà li havea detto che essendo da solo a solo col Cristianissimo, gli havea fatto intendere che mai non havea odiato la persona sua, ancorché havessero hauta così lunga guerra insieme. Hora che lo pregava che se havea a mancarli in cosa alcuna, non lo burlasse nel caso de sua Sorella, che questo reputarebbe a tanta iniuria, che non potrebbe mai scordarsela, né lassar haver in odio la persona sua, e cercar offenderla in ogni cosa che potesse. E dice che 'l Re di Franza li rispose, e con iuramento, voler mantenere quanto havea promesso ${ }^{10}$.

En effet, le rapport de Castiglione sur cet épisode, contenu dans la lettre de Séville du 24 mars 1526 et confirmé par les histoires de Guichardin et de Varchi, nous révèle la complexité des dynamiques politiques à la cour impériale, le développement de plusieurs équilibres de pouvoir et la capacité des différents ministres et factions d'influencer le développement des relations internationales ${ }^{11}$. Même l'empereur semble obligé de prendre en compte la position de son chancelier et, bien qu'il reproche à Gattinara l'appui donné à la cause italienne (à savoir au pape, pour des raisons personnelles, c'est-à-dire afin d'être nommé cardinal), Charles Quint finit par accepter le refus de Mercurino, et même par lui promettre des honneurs ${ }^{12}$. Il est très intéressant d'observer que Castiglione, en rapportant les mots avec lesquels Gattinara se défend de l'accusation d'être partisan du pape et souligne sa fidélité à l'empereur, reproduit un stylème machiavélien : "egli non avea l'occhio ad altro che al servizio di quella (à savoir de sa majesté) ». Dans le chapitre xxII du Prince, Machiavel fait la même suggestion :

$\mathrm{Ma}$, come uno principe possa conoscere el ministro, ci è questo modo, che non falla mai: quando tu vedi el ministro pensare più a sé che a te, e che in tutte le sua azioni vi ricerca dentro l'utile suo, questo tale così fatto mai fia buono ministro, mai te ne potrai fidare. Perché quello che ha lo stato di uno in mano, non debbe pensare mai a sé ma sempre al principe, e non gli ricordare mai cosa che non appartenga a lui ${ }^{13}$.

Ce parallèle nous démontre une fois de plus que la langue de l'ancien chancelier et ambassadeur florentin est mêlée à des expressions typiques du lexique diplomatique et que le lexique politique européen, à partir des années 1520-1530, dépend de la langue de Machiavel.

Dans la correspondance de Castiglione, la langue de la diplomatie nous révèle ses caractéristiques, c'est-à-dire son adaptabilité aux différentes pratiques, le mélange de divers idiomes nationaux, sa réceptivité jusqu'au point d'englober des mots étrangers. Ces aspects sont parfois plus évidents lorsque l'ambassadeur, dans son rapport, s'engage dans la tentative de reproduire avec la plus grande fidélité possible les mots exacts qui ont été utilisés pendant les entretiens et les négociations. Cela est évident dans le compte rendu de l'entretien apparemment privé entre Charles Quint et François I $^{\text {er }}$ (toujours dans la même lettre de Séville du 24 mars 1526), où le mot français méchant et le mot espagnol velliacho pourraient être dérivés, dans la bouche de l'empereur, soit des sources de Castiglione (les diplomates français? le confesseur de Charles Quint ?), soit être simplement le témoignage du mélange linguistique pratiqué à la cour impériale. 
10 Malgré les efforts de Castiglione pour préserver la paix entre le pape et l'empereur, la situation était destinée à se détériorer en l'espace de quelques semaines. Le 23 juin 1526, le pape et les cardinaux envoyèrent en Espagne un bref très sévère où les raisons de l'alliance du pape avec la ligue de Cognac étaient longuement expliquées ainsi que les remontrances contre l'empereur (le texte latin du bref fut rédigé par l'humaniste Jacopo Sadoleto). La cour de Rome s'aperçut très vite que le document envoyé était trop violent, et, le jour suivant, fut rédigé, toujours par Sadoleto, un deuxième bref, bien plus conciliant. Le premier bref arriva en Espagne le 16 août et fut remis par Castiglione à la chancellerie impériale. Le deuxième bref n'arriva en Espagne que le 8 septembre. Le 8 septembre, Castiglione écrivit tout de suite au secrétaire d'État du pape, Nikolaus Schönberg, ainsi qu'au cardinal Giovanni Salviati (ce dernier, cousin de Clément VII et à plusieurs reprises «légat pontifical » en France et en Espagne), en soulignant que les deux brefs avaient été remis à leur destinataire, et qu'il essayerait d'atténuer de toute façon la réaction de l'empereur et de la cour impériale.

In questo punto ho auto lettere di Franza, le quali poco altro contengono che 'l duplicato delle passate, le quali qui mando a V. Sig. Rev.ma, eccetto che in queste l'Arcivescovo [Roberto Acciaiuoli, florentin, nonce à la cour de Louis XII] scrive che' 1 breve mandato prima [c'est-à-dire celui en date 23 juin], la copia del quale è inclusa qui e sigillata B., non si dia. E perché è d'i 24 di giugno, et io l'hebbi alli 16 d'agosto, non pensando che essendo stato tanto tempo in camino dovesse esser rivocato, lo diedi. Hora scriveno ch'el non se dia, et è la lettera scritta el giorno dopo la data del primo breve, ed è giunta hoggi [8 septembre], e data alli 25 di giugno in Roma ${ }^{14}$.

11 En fait, l'empereur et le chancelier Mercurino de Gattinara promirent au nonce de répondre au deuxième bref, mais le 20 septembre Castiglione fut convoqué et reçut une réponse officielle au premier bref. La réponse, rédigée probablement par Alfonso de Valdés, secrétaire chargé de la correspondance en latin auprès de la chancellerie impériale, fut aussi sévère que le bref pontifical du 23 juin ${ }^{15}$.

12 Lorsque Castiglione écrivait le 20 septembre 1526 au secrétaire d'État Schönberg, il ne savait pas qu'à Rome, ce jour même, les pillages avaient déjà commencé et que les milices de Hugo de Moncada et de la famille Colonna avaient obligé le pape à s'enfermer dans le château Saint-Ange. De toute façon, le développement très rapide des événements en Italie (la descente de l'armée du connétable Charles III de BourbonMontpensier aussi bien que sa mort le 6 mai 1527, juste au début de l'assaut à Rome) empêchèrent que la voie de la négociation diplomatique interrompe la guerre et évite le sac de Rome.

13 Après le pillage, Castiglione fut accusé par le pape de ne pas avoir empêché la réaction violente de l'empereur, et notamment d'avoir toujours rassuré la cour de Rome à propos des attitudes de Charles Quint. Castiglione se défendit dans une lettre célèbre du 9 novembre 1527. Du point de vue historiographique, cette lettre n'est pas importante pour la reconstruction des événements, qui sont bien connus grâce à plusieurs sources, mais parce que le nonce souligne encore une fois que seule la stratégie diplomatique est praticable pour la papauté et les États italiens. Impuissante au niveau militaire et des ressources économiques par rapport aux grandes monarchies européennes, la diplomatie italienne ne pouvait se fonder que sur la stratégie de la parole, donc sur un ensemble de pratiques rhétoriques traditionnelles capables de renverser les rapports de force. Cette technique se fonde sur la stratégie de la communication, sur l'habileté à organiser le consensus, sur une connaissance des équilibres politiques et humains à 
l'intérieur des cours et des chancelleries européennes, et enfin, au moins pour le pape, sur un ascendant moral et religieux.

Credo anchorché siano pochi grandi né prelati in Spagna o forse niuno di quelli che in tutto questo tempo sono stati alla corte, ch'io non li habbia sollicitati e stimulati a parlar a l'Imperatore e dolersi delli casi di Vostra Santità acerbamente. [...] Questi sono, Padre Beatissimo, li remedij ch'io ho potuto fare o almen tentare con molto maggior solicitudine [...]. Queste cose, Padre Beatissimo, io mi sono sforzato de imprimerle ne l'animo dell'Imperatore ${ }^{16}$.

C'est justement à ce moment-là que Castiglione conçoit le dessein final de son chefd'œuvre, le dialogue du Courtisan, où il représente une cour parmi les plus petites en Italie, mais il la représente en tant que modèle pour une stratégie politique continentale. Ce serait certainement une erreur de considérer le Courtisan comme un manuel concernant la technique des ambassadeurs ou adressé au chancelier ou bien au fonctionnaire de la cour, ou comme un simple traité de comportement: car le livre ne fonctionne pas sur ce niveau, mais il est un diagnostic de la crise en même temps qu'une proposition afin de véhiculer certains savoirs traditionnels dans le nouveau contexte de la société d'Ancien Régime.

Dans sa lettre au pape du 9 novembre 1527, Castiglione observe que pendant les mois de son activité en tant que nonce en Espagne, il a toujours essayé de créer un climat favorable au pape auprès de la cour impériale, en sollicitant les "grands" (l'aristocratie) et les prélats pour qu'ils manifestent leur désappointement à l'empereur devant la situation du pape. À la fin de la lettre, Castiglione souligne que cette stratégie, c'est-à-dire une stratégie presqu'exclusivement rhétorique, était le seul remède qu'il a pu tenter. Déjà le mot « remède » nous amène dans l'univers conceptuel machiavélien, où la politique est conçue justement en tant qu'art du remède, en tant que technique médicale afin de soigner un organisme (la république) malade.

Juste après le sac, Castiglione sera engagé à reconstruire les négociations diplomatiques entre la papauté et l'Empire, jusqu'à cette ligne d'arrivée constituée par la paix de Bologne de 1530, accord qui aurait valu à Castiglione sa nomination comme cardinal, s'il n'était mort malheureusement un an auparavant. Dans cette nouvelle phase d'activité pour le nonce, on relève la violente querelle avec Alfonso de Valdés ${ }^{17}$. Alfonso était très proche des idées d'Érasme et suivait avec attention leur circulation en Espagne (tout comme son frère Juan) : il avait d'abord diffusé la réponse de l'empereur au bref pontifical de juin 1526 avec d'autres pamphlets favorables à l'empereur à travers une édition imprimée au printemps 1527 (Pro divo Carolo apologetici libri duo). Au début de septembre 1528, lorsque la cour impériale était à Burgos, Alfonso composa un dialogue imaginaire, Diálogo de las cosas acaecidas en Roma, où il attribuait au sac de Rome la valeur d'un événement prédestiné par le jugement divin contre la corruption de l'Église, et à la politique de Charles Quint la dimension d'une action messianique déterminée par la nécessité de garder l'unité des chrétiens ${ }^{18}$. De plus, il adressa à Castiglione une lettre concernant son dialogue, qui est une véritable excusatio non petita; en réponse à cette lettre d'Alfonso, Castiglione écrivit et diffusa une longue lettre très violente contre de Valdés. En soulignant le caractère hérétique des argumentations de Valdés, le but de Castiglione était de discréditer Alfonso face à ses propres patrons (le chancelier Mercurino et l'empereur lui-même). La stratégie de Castiglione n'eut qu'un succès partiel : en effet, Alfonso ne fut pas éloigné de la cour impériale ni condamné pour ses affirmations, et il resta dans la chancellerie jusqu'à sa mort, pendant l'épidémie de peste en 1532, lorsque Charles Quint était engagé sur le 
front hongrois. Cette lettre de Castiglione est le premier témoignage d'une stratégie idéologique qui caractérisera la deuxième moitié $d u \mathrm{XVI}^{\mathrm{e}}$ siècle et tout le $\mathrm{XVII}^{\mathrm{e}}$, à savoir la superposition entre l'accusation d'hérésie et celle d'infidélité politique, entre le crime contre l'État et contre la majesté de l'Église, jusqu'à reconstruire le délit de maiestas, en tant que nouvelle et pénétrante typologie de contrôle des consciences politiques et religieuses ${ }^{19}$.

17 Au moment même où se déroule la querelle avec de Valdés, Castiglione est engagé dans la diffusion du Courtisan, où il propose un modèle ambigu, toujours en équilibre entre l'apparence et la vérité, mais si possible capable de nager dans les eaux périlleuses des cours d'Ancien Régime. En somme, il offre une formation pour le gentilhomme qui lui permette de revendiquer un rôle de conseiller du prince.

Il fin adunque del perfetto cortegiano, del quale insino a qui non s'è parlato, estimo io che sia il guadagnarsi per mezzo delle condicioni attribuitegli da questi signori talmente la benevolenza e l'animo di quel principe a cui serve, che possa dirgli e sempre gli dica la verità d'ogni cosa che ad esso convegna sapere, senza timor o periculo di dispiacergli ${ }^{20}$.

Pendant la vie de Castiglione, nous n'assistons pas à la transformation des équilibres de pouvoir en tant que tels, mais plutôt à une métamorphose des compétences (et pour cette raison à une modification profonde des statuts éducatifs et des modèles). La recherche de nouvelles formes concerne aussi bien le plan juridique que le plan de la rhétorique: la littérature est appelée à rendre évidentes et reconnaissables les nouvelles structures socio-politiques. C'est dans cet espace complexe que le Courtisan gagne son rôle de livre de chevet pour la formation des élites dirigeantes européennes, notamment grâce à son ambiguïté, à la dialectique interne entre l'être et le devoir être : le chef-d'œuvre de Castiglione offre une visibilité à ce qui reste implicite dans ses pages, à savoir les nouvelles pratiques du gouvernement ${ }^{21}$.

Le courtisan est donc le véritable remède à l'ignorance des princes. L'œuvre de Castiglione manque d'une analyse et d'un véritable discours sur les techniques et les outils du gouvernement: aussi, la distance entre la réalité historique (tant sur l'incapacité que sur la lâcheté des princes italiens, au cœur du propos de Guicciardini ou de Machiavel) et l'élan du projet politique semble-t-elle configurer une dimension utopique ou bien confiner le Courtisan dans l'espace de la réflexion philosophique entre le rattachement aux modèles aristotéliciens et plutarquiens et la version courtisane des thèses néoplatoniciennes de Ficin. Néanmoins, Castiglione sait pertinemment que le roi ne veut pas être un philosophe et que le philosophe ne peut pas devenir roi: l'expérience de Castiglione lui a montré que le courtisan ne devra pas se limiter à former le prince, mais grâce au dialogue, par le biais de la conversation, il devra obtempérer à une obligation de vérité, faire en sorte «che possa dirgli e sempre gli dica la verità d'ogni cosa che ad esso convegna sapere ».

Castiglione nous propose ici une conception érasmienne de l'éducation du prince et même de la relation entre le courtisan et le prince, jusqu'à souligner que "certo è che l'animo di colui che pensa di far che 'l suo principe non sia d'alcuno ingannato, né ascolti gli adulatori, né i maledici e bugiardi e conosca il ben, e 'l male ed all'uno porti amore, all'altro odio, tende ad ottimo fine ${ }^{22}$ ». "Flatteurs, médisants, menteurs »: il s'agit précisément des catégories contre lesquelles Erasme menait sa polémique: "Sed est omnium pestilentissimum adulatorum genus, qui specie libertatis adsentantur ac miro quodam artificio, 
dum repugnant impellunt, dum obiurgant laudant. Hos mire depinxit Plutarchus in libello, cui titulum fecit: Quomodo dignosci possit amicus ab adulatore ${ }^{23}$. »

21 La capacité du courtisan à soigner l'ignorance de son prince est représentée par Castiglione grâce à une métaphore lucrétienne (qui sera reprise par Bernardo Tasso et ensuite par Torquato Tasso au début de la Jérusalem délivrée) :

In questo modo per la austera strada della virtù [il cortegiano] potrà condurlo [il principe], quasi adornandola di fronde ombrose e spargendola di vaghi fiori [...], ingannandolo con inganno salutifero come i cauti medici, li quali spesso, volendo dar a fanciulli infermi e troppo delicati medicina di sapore amaro, circondano l'orificio del vaso di qualche dolce liquore ${ }^{24}$.

Les pages du Courtisan relient les causes de la catastrophe politique italienne (à savoir la crise militaire, économique et politique) à la crise morale des États italiens et à l'incapacité des princes. Castiglione nous parle à partir d'un horizon de décadence, qui constitue une limite à l'action politique. En transférant son expérience biographique dans son œuvre, l'auteur ne reste pas au-dessus de la mêlée, mais il cherche des solutions; sa voix participe au débat, désordonné et confus, dans lequel plusieurs parcours possibles se chevauchent dans une dynamique historique complexe. La conclusion du dialogue n'est pas une célébration vide du passé, mais une ouverture vers le scénario d'une transformation rapide de la modernité, la dimension de l'homme d'État dans les futures villes du monde.

\section{BIBLIOGRAPHIE}

BURKE Peter, Le fortune del Cortegiano. Baldassarre Castiglione e i percorsi del Rinascimento europeo, traduit en italien par A. Merlino, Rome, Donzelli, 1998, p. 4-39.

CASTIGLIONE Baldassarre, Le livre du Courtisan, traduit de l'italien d'après la version de G. Chappuis (1580), par A. Pons, Paris, Lebovici, 1987 (ensuite, Paris, Flammarion, 1991).

CASTIGLIONE Baldassarre, Il Libro del Cortegiano, 1. La prima edizione nelle case d'Aldo Romano e d'Andrea d'Asola suo suocero, Venezia, aprile 1528 ; 2 . Il manoscritto di tipografia L: Biblioteca Medicea Laureanziana, Ashburnhamiano 409 ; 3. L'autore (e i suoi copisti), l'editore, il tipografo. Come il Cortegiano divenne libro a stampa. Nota ai testi di L e Ad., A. Quondam (éd.), Rome, Bulzoni, 2016.

CASTIGLIONE Baldassarre, Lettere famigliari e diplomatiche, G. La Rocca, A. Stella et U. Morando (éds), avec la Lettera ad Alfonso Valdés, éditée par P. Pintacuda, une note philologique par R. Vetrugno, et une note pour les images par L. Bianco, Turin, Einaudi, 2016, 3 vol.

Correspondenz des Kaisers Karl V, aus dem königlichen Archiv und der Bibliothèque de Bourgogne zu Brüssel mitgetheilt von Karl Lanz, Leipzig, Brockhaus, 1844, vol. I (1513-1532), p. 217 et 219-221.

D’Amico Juan Carlos, « Castiglione, Érasme et Plutarque : le "prince parfait" et la "patrie universelle" entre mythes et réalités ", dans P. Grossi et J. C. D’Amico (éds), De la politesse à la politique. Recherches sur les langages du Livre du Courtisan, Actes du colloque de l'université de Caen (2000), Presses universitaires de Caen, 2001, p. 121-151. 
ÉRASME, L'éducation du prince chrétien, traduit du latin par A.-M. Greminger et présenté par J.-C. Saladin, Paris, Les Belles Lettres, 2016.

FEDELE Dante, «Dire la vérité au prince : le Livre du Courtisan de Baldassarre Castiglione », dans M. Abbès et D. Ottaviani (dir.), Philosophie politique médiévale et émergence de la modernité : Orient/ Occident, Paris, Classiques Garnier, 2018 (à paraître).

FOURNEL Jean-Louis, « Savoir vivre et savoir faire dans le Livre du Courtisan de Baldassar Castiglione (1528) : les aléas de la mondanité et de la politique ", dans The Russian Imperial Court and Europe: Dialogues of Cultures, Transactions of the State Hermitage, XXVI, Actes du colloque de Saint-Pétersbourg (18-21 octobre 2005), Saint-Pétersbourg, 2008, p. 79-94.

FoURNEL Jean-Louis, « Mantova, agosto 1530. La cortina di fumo del classicismo », dans Atlante della letteratura italiana, vol. 2, Turin, Einaudi, 2011, p. 8-13.

Jacobi Sadoleti, Epistolae Leonis X, Clementis VII, Pauli III nomine scriptae, accessit Antonii Florebelli de vita eiusdem Sadoleti, commentarius et epistolarum liber, Romae, Salomonius, 1759, p. 161-173 et p. 173-174.

LONGHURST John E., Alfonso de Valdés and the Sack of Rome. Dialogue of Lactancio and an Archdeacon, Albuquerque, New Mexico Univ. Press, 1952.

MACHIAVEL, De principatibus. Le Prince, traduction et commentaire de J.-L. Fournel et J.-C. Zancarini, texte italien établi par G. Inglese, Paris, PUF, 2014 (2000).

Pro divo Carolo [...] apologetici libri duo nuper ex Hispania allati, Antverpiae, apud Dumaeum Godfridum, 1527, p. 9-92.

SBRICCOLI Mario, "Crimen laesae maiestatis". Il problema del reato politico alle soglie della scienza penalistica moderna, Milan, Giuffré, 1974.

SCARPATI Claudio, « Dire la verità al principe (sulle redazioni di Cortegiano, IV, 4-48) », Aevum, vol. 57, n 3, 1983, p. 428-449.

VALDÉs Alfonso DE, Diálogo de la cosas ocurridas en Roma, J. F. Montesinos (éd.), Madrid, EspasaCalpe, $1956\left(1928^{1}\right)$.

VALDÉs Alfonso DE, Due dialoghi, avec une traduction italienne du XVI ${ }^{\mathrm{e}}$ siècle, G. De Gennaro (éd.), Naples, Ist. universitario orientale, 1968.

\section{NOTES}

1. B. Castiglione, Il Libro del Cortegiano, 1. La prima edizione nelle case d'Aldo Romano e d'Andrea d'Asola suo suocero, Venezia, aprile 1528 ; 2 . Il manoscritto di tipografia L: Biblioteca Medicea Laurenziana, Ashburnhamiano 409 ; 3 . L'autore (e i suoi copisti), l'editore, il tipografo. Come il Cortegiano divenne libro a stampa. Nota ai testi di L e Ad., A. Quondam (éd.), Rome, Bulzoni, 2016. Édition traduite par A. Pons d'après la version de G. Chappuis (1580), Paris, Lebovici, 1987 (Flammarion, 1991).

Pour les citations on indiquera aussi bien la division traditionnelle en chapitres (Cian) que la division moderne en paragraphes (Quondam).

2. P. Burke, Le fortune del Cortegiano. Baldassarre Castiglione e i percorsi del Rinascimento europeo, traduit en italien par A. Merlino, Rome, Donzelli, 1998, p. 4-39.

3. B. Castiglione, Il Libro del Cortegiano, ouvr. cité, dedica 14.

4. R. Ruggiero, Baldassarre Castiglione diplomatico. La missione del cortegiano, Florence, Olschki, 2017, p. 107-34. 
5. A. Quondam, L'autore (e i suoi copisti), ouvr. cité, p. 50-54.

6. J.-L. Fournel, «Savoir vivre et savoir faire dans le Livre du Courtisan de Baldassar Castiglione (1528) : les aléas de la mondanité et de la politique », dans The Russian Imperial Court and Europe: Dialogues of Cultures, Transactions of the State Hermitage, XXVI, Actes du colloque de Saint-Pétersbourg (18-21 octobre 2005), Saint-Pétersbourg, 2008, p.79-94 et Id., "Mantova, agosto 1530. La cortina di fumo del classicismo», dans Atlante della letteratura italiana, vol. 2, Turin, Einaudi, 2011, p. 8-13.

7. B. Castiglione, Lettere famigliari e diplomatiche, G. La Rocca, A. Stella et U. Morando (éds), avec la Lettera ad Alfonso Valdés, éditée par P. Pintacuda, une note philologique par R. Vetrugno, et une note pour les images par L. Bianco, Turin, Einaudi, 2016, 3 vol. (voir le compte rendu de cette édition par R. Ruggiero, Laboratoire italien, 2017).

8. R. Ruggiero, Baldassare Castiglione diplomatico, ouvr. cité, p. 47-58.

9. N. Machiavel, De principatibus. Le Prince, traduction et commentaire de J.-L. Fournel et J.-C. Zancarini, texte italien établi par G. Inglese, Paris, PUF, 2014 (2000), chap. XI , § 2-4.

10. B. Castiglione, Lettere, ouvr. cité, t. 3 , lettre $n^{\circ} 1699$, p. 211-213: à Nikolaus Schönberg, archevêque de Capua et secrétaire d'État à la cour pontificale, de Séville le 24 mars 1526.

11. F. Guicciardini, Storia d'Italia, S. Seidel-Menchi (éd.), Turin, Einaudi, 1971, livre XVI, chap. XV ; B. Varchi, Storia fiorentina, L. Arbib (éd.), Florence, Società editrice delle storie del Nardi e del Varchi, 1838-1841, livre II, t. 1, p. 89.

12. M. Arborio di Gattinara, Autobiografia, traduite du latin avec commentaire par G. Boccotti, Rome, Bulzoni, 1991, p. 106-107 et 120-128 ; J. C. D’Amico, « Gattinara et la monarchie impériale de Charles Quint. Entre millénarisme, translatio imperii et droits du Saint-Empire », Asterion, $\mathrm{n}^{\circ} 10$, 2012.

13. N. Machiavel, De principatibus. Le Prince, ouvr. cité, chap. XXII, § 6-7.

14. B. Castiglione, Lettere, ouvr. cité, t. 3 , lettre $n^{\circ} 1724$, p. 265 : au card. Giovanni Salviati, de Grenada, le 8 septembre 1526. Le texte des deux brefs pontificaux (les deux lettres envoyées à la cour impériale le 23 et le 25 juin 1526) est dans le recueil Jacobi Sadoleti, Epistolae Leonis X, Clementis VII, Pauli III nomine scriptae, accessit Antonii Florebelli de vita eiusdem Sadoleti, commentarius et epistolarum liber, Rome, Salomonius, 1759, p. 161-173 et p. 173-174.

15. Pro divo Carolo [...] apologetici libri duo nuper ex Hispania allati, Antverpiae, apud Dumaeum Godfridum, 1527, p. 9-92. Voir K. Lanz, Correspondenz des Kaisers Karl V, aus dem königlichen Archiv und der Bibliothèque de Bourgogne zu Brüssel mitgetheilt von Karl Lanz, Leipzig, Brockhaus, 1844, vol. I (1513-1532), p. 217 et 219-221.

16. B. Castiglione, Lettere, ouvr. cité, t. 3, lettre $n^{\circ} 1767$, p. 363-364 : à Clément VII, de Burgos, le 9 novembre 1527.

17. R. Ruggiero, Baldassare Castiglione diplomatico, ouvr. cité, p. 99-106. Aussi bien la lettre de Valdés à Castiglione que la sévère réponse de ce dernier sont maintenant dans l'édition citée de Castiglione, Lettere famigliari e diplomatiche, t. 3, p. 625-701.

18. A. de Valdés, Diálogo de la cosas ocurridas en Roma, J. F. Montesinos (éd.), Madrid, Espasa-Calpe, 1956 (1928 $)$; J. E. Longhurst, Alfonso de Valdés and the Sack of Rome. Dialogue of Lactancio and an Archdeacon, Albuquerque, New Mexico Univ. Press, 1952; A. de Valdés, Due dialoghi, avec une traduction italienne du XvI ${ }^{\mathrm{e}}$ siècle, G. De Gennaro (éd.), Naples, Ist. universitario orientale, 1968.

19. Voir M. Sbriccoli, Crimen loesce maiestatis. Il problema del reato politico alle soglie della scienza penalistica moderna, Milan, Giuffré, 1974.

20. B. Castiglione, Il Cortegiano, ouvr. cité, IV V = IV II 15.

21. D. Fedele, «Dire la vérité au prince : le Livre du Courtisan de Baldassarre Castiglione », dans M. Abbès et D. Ottaviani (dir.), Philosophie politique médiévale et émergence de la modernité: Orient/ Occident, Paris, Classiques Garnier, 2018 (à paraître).

22. B. Castiglione, Il Cortegiano, ouvr. cité, IV V = IV II 19. Voir C. Scarpati, «Dire la verità al principe (sulle redazioni di Cortegiano, IV, 4-48) », Ævum, vol. 57, n 3, 1983, p. 428-449. 
23. Érasme, L'éducation du prince chrétien, traduit du latin par A.-M. Greminger et présenté par J.C. Saladin, Paris, Les Belles Lettres, 2016, p. 128-129 : « Mais la plus nocive de toutes les espèces de flatteurs, c'est celle qui, sous couvert de liberté et par une sorte d'artifice, encourage en s'opposant et loue en critiquant. Plutarque en a donné une description remarquable dans l'opuscule dont voici le titre : Comment distinguer l'ami du flatteur. » Cf. J. C. D’Amico, « Castiglione, Érasme et Plutarque : le "prince parfait" et la "patrie universelle" entre mythes et réalités ", dans P. Grossi et J. C. D’Amico (éds), De la politesse à la politique. Recherches sur les langages du Livre du Courtisan, Actes du colloque de l'université de Caen (2000), Presses universitaires de Caen, 2001, p. 121-151.

24. B. Castiglione, Il Cortegiano, ouvr. cité, IV X = IV II 43-44. Le Tasse, La Jérusalem délivrée, I III : "Così à l'egro fanciul porgiamo aspersi / di soavi licor gli orli del vaso: / succhi amari ingannato intanto ei beve, / e da l'inganno suo vita riceve. »

\section{RÉSUMÉS}

Les dernières années de la vie de Castiglione furent celles de son activité de nonce pontifical à la cour impériale de Charles Quint en Espagne, juste avant et juste après le sac de Rome. La correspondance de Castiglione avec la cour de Rome et le pape Clément VII constitue un véritable miroir des pratiques politiques et diplomatiques italiennes pendant les années des guerres d'Italie et de la crise européenne.

Gli ultimi anni della vita di Castiglione furono impegnati dalla nunziatura apostolica presso la corte imperiale di Carlo V in Spagna, nelle fasi della guerra franco-spagnola che precedettero e seguirono il sacco di Roma. Le lettere di Castiglione con la curia romana e papa Clemente VII costituiscono un efficace specchio delle procedure diplomatiche e della politica italiana negli anni delle guerre d'Italia e della crisi europea.

Castiglione spent the last years of his life as "nuntius apostolicus" (that is permanent ambassador of the pope) at the imperial court of Charles $\mathrm{V}$ in Spain, during the period just before and immediately after the Sack of Rome. Castiglione's letters to the papal court at Rome and directly to Clement VII were an effective mirror of Italian politics and diplomacy during the Renaissance Italian wars and the European crisis.

\section{INDEX}

Parole chiave : Baldassarre Castiglione, sacco di Roma, diplomazia

Keywords : Baldassarre Castiglione, Sack of Rome, diplomacy

Mots-clés : Baldassarre Castiglione, sac de Rome, pratiques diplomatiques 
AUTEUR

RAFFAELE RUGGIERO

Aix-Marseille Université, CAER, Aix-en-Provence, France

raffaele.ruggiero@univ-amu.fr 